\title{
Cardiac telocytes as principal interstitial cells for myocardial reparation and regeneration after infarction - our hope
}

\author{
Varga $\mathrm{I}^{1}$, Kyselovic $\mathrm{J}^{2}$, Danihel $\mathrm{L}^{3}$, Klein $\mathrm{M}^{1}$, Barczi $\mathrm{T}^{4}$, Galfiova $\mathrm{P}^{1}$, Danisovic $\mathrm{L}^{5}$ \\ Institute of Histology and Embryology, Faculty of Medicine, Comenius University in Bratislava, \\ Slovakia.ivan.varga@fmed.uniba.sk
}

\section{ABSTRACT}

According to our knowledge, this is the first research experiment that focuses on the study of the distribution of c-kit positive cells at the sites of myocardial infarction in human hearts (Fig. 3, Ref. 16). Text in PDF www.elis.sk. KEY WORDS: cardiac telocytes.

The human body consists of approximately 200 types of morphologically and functionally different cells. The number of different types of cells will be probably never known exactly, because some cells may be in different stages of development. Only a few years ago, a research team led by professor Laurentiu Popescu from Bucharest, Romania described a completely new cell population. These newly discovered cells were first referred to as interstitial Cajal-like cells. This name was reflecting their apparent resemblance to the interstitial cells of Cajal, pacemaker cells in the wall of gut observed by Santiago Ramón y Cajal more than 100 years ago. As further studies gradually exposed their unique ultrastructure and immunophenotype, it became obvious, that interstitial Cajal-like cells are different from gastrointestinal interstitial cells of Cajal. Therefore, it was proposed to call these new cells telocytes (1).

Very interesting is to study the functional roles of telocytes in different organs, especially from the point of view of regenerative medicine. The question of telocytes is one of the most attractive questions in histology and regenerative medicine in the last five years. The processes of regeneration and reparation in the heart, the most important vital organ of the human body, are not only fascinating from the scientific point of view, but are also clinically very important. For this reason the article entitled “A comprehen-

${ }^{1}$ Institute of Histology and Embryology, Faculty of Medicine, Comenius University in Bratislava, Slovakia, ${ }^{2} 1$ st Department of Internal Medicine, Faculty of Medicine, Comenius University in Bratislava, Slovakia, ${ }^{3}$ Institute of Pathology, Faculty of Medicine, Comenius University in Bratislava, Slovakia, ${ }^{4}$ Institute of Anatomy, Faculty of Medicine, Comenius University in Bratislava, Slovakia, and ${ }^{5}$ Institute of Medical Biology, Genetics and Clinical Genetics, Faculty of Medicine, Comenius University in Bratislava, Slovakia

Address for correspondence: I. Varga, RND, PhD, Institute of Histology and Embryology, Faculty of Medicine, Comenius University in Bratislava, Sasinkova 4, SK-811 08 Bratislava, Slovakia.

Phone: +421.2.59357547

Acknowledgment: This study was supported by Grant of the Slovak Research and Development Agency No. APVV-0434-12 "Morphological characterization of reparative and regenerative mechanisms in myocardium during chronic diseases” and Grant No. APVV-14-0032. sive guide to telocytes and their great potential in cardiovascular system”, published by the Bratislava Medical Journal (2) has brought us an optimistic expectations, that telocytes will make the myocardial regeneration possible in near future. We share, in our recently published article (3), some of the cited authors' view especially on the role of cardiac telocytes as the key cell population responsible for the maintenance of the cardiac microenvironment homeostasis, by the means of functional interconnection and integration of a wide variety of cell types within the heart (cells of immune system, endothelial cells, cardiomyocytes, stem cells, etc.). However, we do not support the confidence of data about molecular biology, morphology, and pathophysiology of telocytes published by Kucybala et al (2) because of very simplistic and unadvised presentation. Application of results from experimental studies into clinical practice has to be very careful and responsible.

It is necessary to point out, that there is an ongoing wide dispute among histologists, pathologists and cell biologists about the nature of telocytes as a distinct and unique cell population. One of the principal opinions, opposing their status as an individual cell type, is that they represent "only" a highly specialized population/subtype of fibroblasts, endothelial cells, or immunological stem cells. Because of such arguments a broad consensus is still kept out of reach. It has not been changed even by the fact, that PubMed database reads more than 240 publications after key word "telocytes" is typed into the search box and more than $50 \%$ of the results are from the last three years. Aforementioned discrepancy is well documented in the works of Díaz-Flores et al (4), who termed this cell population as "CD34+ stromal fibroblastic cells," Ivey and Tallquist (5) who used "cardiac telocyte” as a synonymic term for "cardiac fibroblast”, but also in the newest hypothesis published by Rusu et al (6) who described some subsets of cardiac telocytes as cells belonging to endothelial lineage.

The processes of regeneration and reparation after injuries and during chronic diseases modify tissue architecture by a sequential orchestration of events including cell proliferation, differentiation, dedifferentiation and cell apoptosis or necrosis (7). During evaluation of the results focused on the role of telocytes, it has to 

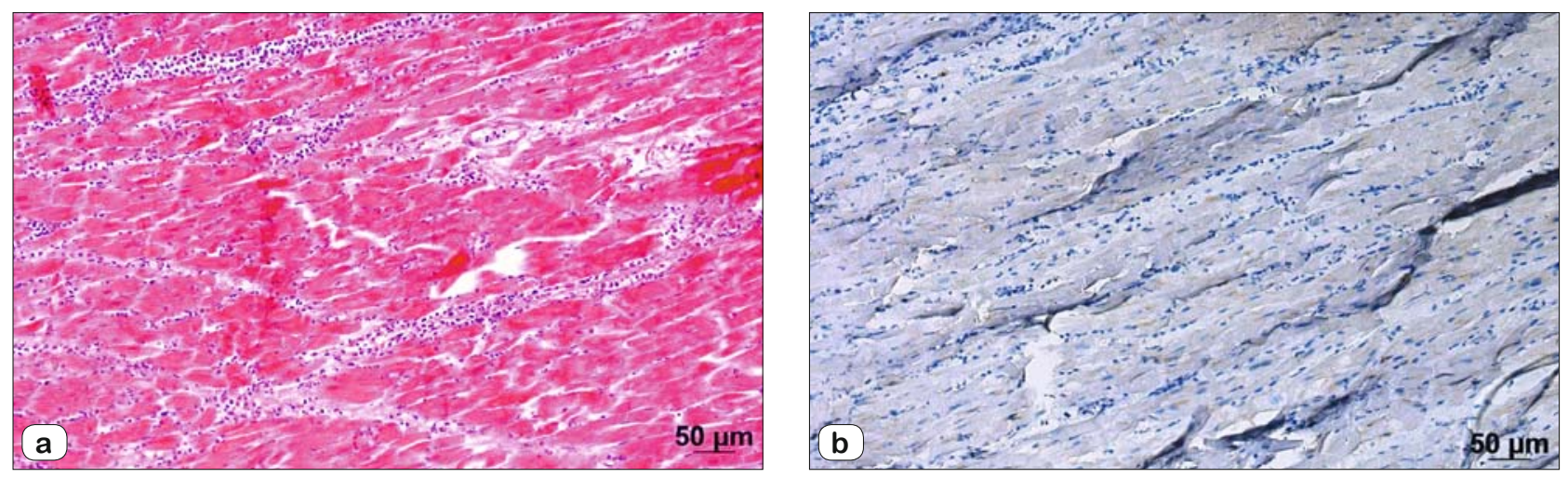

Fig. 1. Acute myocardial infarction, 68-years-old male. a) Microscopy image (hematoxylin and eosin staining) from autopsy specimen of myocardial infarction. The myocardium shows pale and fragmented cardiomyocytes with loss of nuclei and markedly decreased cross-striations; prominent infiltration by polymorphonuclear leukocytes is visible. b) No c-kit (CD117)-positive cells are detected between cardiac muscle cells (orig. magn. 200x).
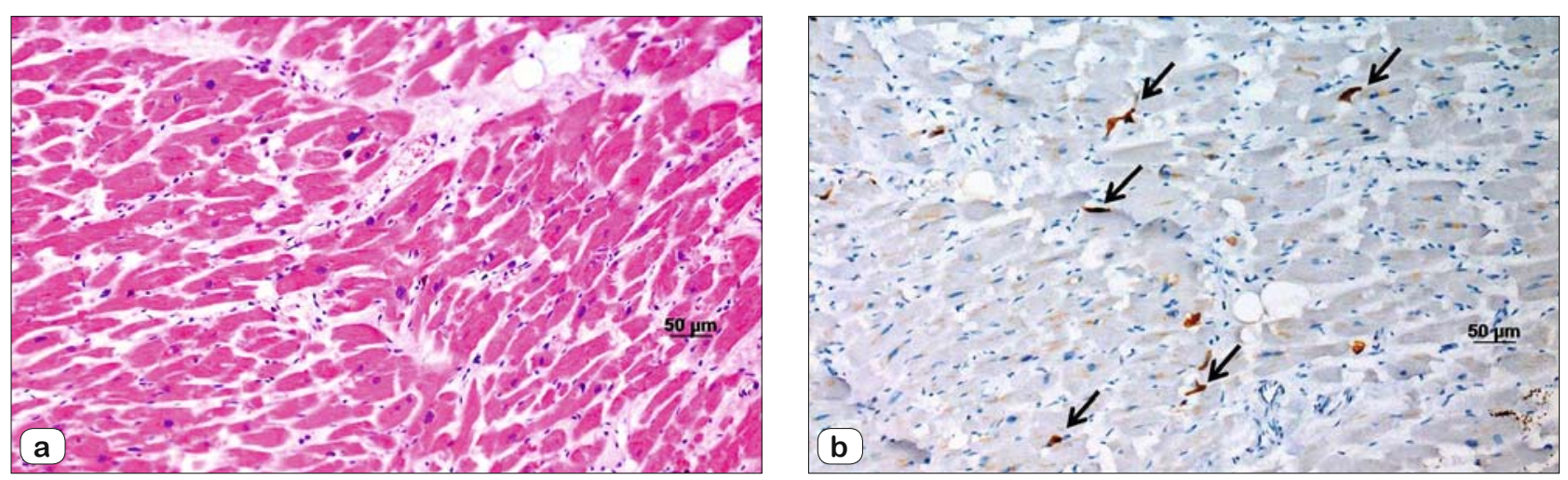

Fig. 2. Normal myocardium, 67-years-old female. a) histologically normal myocardium (hematoxylin and eosin staining). b) Spindle-shaped c-kit positive cells, probably telocytes (arrows), between cardiac muscle cells (orig. magn. 200x).
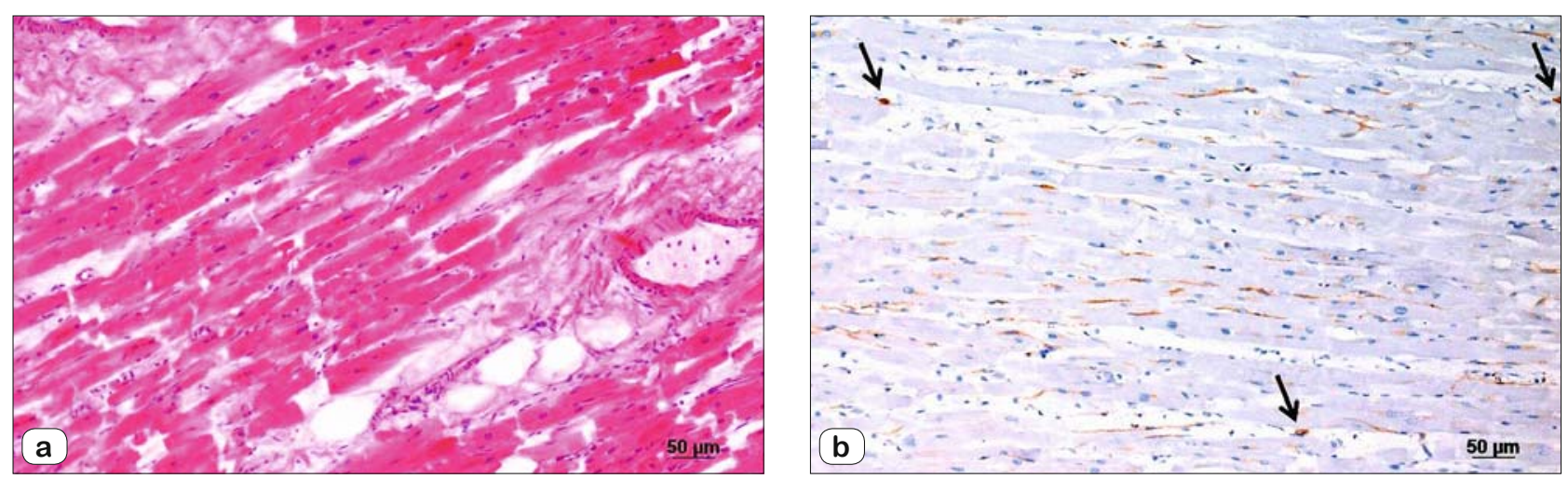

Fig. 3. Myocardium with small scars after previous hypoxia, 57-years-old male. a) Histologically normal cardiac muscle cells between increased perivascular connective tissue (hematoxylin and eosin staining). b) Numerous spindle-shaped c-kit positive cells with long prolongations, probably telocytes (brown color) between cardiac muscle cells. Arrows indicated c-kit positive round cells, probably mastocytes (orig. magn. 200x).

be clearly stated which results were achieved from experimental, animal and human studies. This is also a problem of Kucybala's et al review (2). It is very hopeful that some recently published studies investigated changes in the distribution and function of telocytes associated with cardiovascular disease. But most of them are based on experiments on rats (8-10) and only one paper is focused on the investigation of human end-stage failing hearts (11). How- ever, to describe cardiac telocytes as having therapeutic potential for humans inappropriate at this stage of research, because there are too many controversies. The same applies to cardiac telocytes experimental transplantation in animal models which significantly increase vessel density and decrease myocardial fibrosis at the heart infarction site (9). Up to now, the distribution of telocytes within the infarction site and scar tissue in the human heart is still unknown. 
Our preliminary study was focused on the immunohistochemical detection of telocytes at the site of acute myocardial infarction, as well as at different times after the infarction. Myocardial tissue was obtained during necropsies of a group consisting of 20 human hearts (mean age $70.9 \pm 11.2$ ). The immunohistochemical characterization of human cardiac telocytes was performed using a c-kit (CD117) antibody (Dako, Denmark). However, it has to be mentioned that antibodies against c-kit (CD117) are not telocyte-specific, they label the transmembrane tyrosine kinase receptor located on the surface of wide variety of cells (e.g. stem cells and mast cells). Primary antibodies were used according to the manufacturer's protocols. For visualization, we used EnVision $^{\mathrm{TM}}$ FLEX Detection system (HRP SM802, Dako, Denmark) with diaminobenzidine as a chromogen. Cell nuclei were stained with Mayer's hematoxylin. Our preliminary results are represented by the photomicrographs $1-3$. Cells, positive for c-kit, probably telocytes, were not detected at the site of acute myocardial infarction (Fig. 1). We assume that they perished as a result of the infarction development, since telocytes are typically found at the sites of the myocardium unaffected by necrosis. In contrast with the aforementioned experiments on rats, the number of c-kit positive spindle-shaped telocytes in human myocardium from patients who have overcame the heart attack, varies significantly. In some cases, we truly found a higher number of telocytes inside myocardium nearby the site of connective tissue scars (Fig. 2 and 3), while in other cases, we couldn't detect almost none of these cells.

However, it's important to note, that the research of telocytes in human hearts is markedly different from the research layout in animal models. Firstly, the human patient age, as the main contributor to diminished regenerative capacity of the whole organism, is relatively higher. Furthermore, the infarction sites are localized in various areas within the heart. This situation is in a sharp contrast to what can be achieved in rats - the rat model with a surgically induced infarction by the means of ligation of the left anterior descending coronary artery, creates the possibility to target the same specific selected part of the myocardium. Moreover, the treatment of the patients with the acute myocardial infarction is not always the same, as different drugs affecting the heart and metabolism are often used. It is also necessary to point out, that elderly patients usually suffer from multiple comorbidities. All these unstable factors in humans may influence the morphology of cardiovascular system. In spite of the mentioned variables, we still agree, that the observations in animal models may suggest that telocytes play an important role in the regeneration/reparation of the myocardium, or in the neoangiogenesis at the sites affected by myocardial infarction. We do not like to degrade their importance. On the other hand, we must emphasize, that the further research into the role of telocytes in similar reparative mechanisms within the human myocardium, is still necessary.

According to our knowledge, this is the first research experiment that focuses on the study of the distribution of c-kit positive cells at the sites of myocardial infarction in human hearts. The pathophysiological consequences of altered telocytes' number in many heart diseases are largely unknown (12). On the other hand, the role of cardiac telocytes as progenitor cells and / or "nurse cells" (support- ing cells) in the human endocardial stem cell niches were described in numerous studies $(13,14)$. We incline to the hypothesis of other authors that, in the future, telocytes might have a huge therapeutic potential for the design of some future cell-based cardiac regeneration, repair and protection strategies $(15,16)$. But the way from experimental animal studies to clinically important results, which may directly improve the quality of life of patients with different acute and chronic cardiac diseases, is extremely long and demanding.

\section{References}

1. Popescu LM, Faussone-Pellegrini M-S. Telocytes - a case of serendipity: the winding way from Interstitial Cells of Cajal (ICC), via Interstitial Cajal-Like Cells (ICLC) to telocytes. J Cell Col Med 2010; 14 (4): 729-740.

2. Kucybala I, Janas P, Ciuk S, Cholopiak W, Klimek-Piotrowska W, Holda MK. A comprehensive guide to telocytes and their great potential in cardiovascular system. Bratisl Lek Listy 2017; 118 (5): 302-309.

3. Varga I, Danisovic L, Kyselovic J, Gazova A, Musil P, Miko M, Polak S. The functional morphology and role of cardiac telocytes in myocardium regeneration. Can J Physiol Pharmacol 2016; 94 (11): 1117-1121.

4. Díaz-Flores L, Gutiérrez R, García MP, Sáez FJ, Díaz-Flores L Jr, Valladares F, Madrid JF. CD34+ stromal cells/fibroblasts/fibrocytes/telocytes as a tissue reserve and a principal source of mesenchymal cells. Location, morphology, function and role in pathology. Histol Histopathol 2014; 29 (7): 831-870.

5. Ivey MJ, Tallquist MD. Defining the cardiac fibroblast. Circ J 2016; 80(11): 2269-2276.

6. Rusu MC, Hostiuc S, Vrapciu AD, Mogoantă L, Mănoiu VS, Grigoriu F. Subsets of telocytes: Myocardial telocytes. Ann Anat 2017; 209: 37-44.

7. Uygur A, Lee RT. Mechanisms of cardiac regeneration. Dev Cell 2016; 36 (4): 362-374.

8. Xiao J, Chen P, Qu Y, Yu P, Yao J, Wang H, Fu S, Bei Y, Chen Y, Che $\mathbf{L}, \mathbf{X u} \mathbf{J}$. Telocytes in exercise-induced cardiac growth. J Cell Mol Med 2016; 20 (5): 973-979.

9. Zhao B, Liao Z, Chen S, Yuan Z, Yilin C, Lee KK, Qi X, Shen X, Zheng X, Quinn T, Cai D. Intramyocardial transplantation of cardiac telocytes decreases myocardial infarction and improves post-infarcted cardiac function in rats. J Cell Mol Med 2014; 18 (5): 780-789.

10. Manole CG, Cismaşiu V, Gherghiceanu M, Popescu LM. Experimental acute myocardial infarction: telocytes involvement in neo-angiogenesis. J Cell Mol Med 2011; 15(11): 2284-2296.

11. Richter M, Kostin S. The failing human heart is characterized by decreased numbers of telocytes as result of apoptosis and altered extracellular matrix composition. J Cell Mol Med. 2015; 19 (11): 2597-2606.

12. Kostin S. Cardiac telocytes in normal and diseased hearts. Sem Cell Dev Biol 2016; 55: 22-30.

13. Grigoriu F, Hostiuc S, Vrapciu AD, Rusu MC. Subsets of telocytes: the progenitor cells in the human endocardial niche. Rom J Morphol Embryol 2016; 57 (Suppl 2): 767-774.

14. Bei Y, Wang F, Yang C, Xiao J. Telocytes in regenerative medicine. J Cell Mol Med 2015; 19 (7): 1441-1454.

15. Bei Y, Zhou Q, Sun Q, Xiao J. Telocytes in cardiac regeneration and repair. Semin Cell Dev Biol 2016; 55: 14-21.

16. Fu S, Zhu H, Li S, Wang Y, Bei Y, Xiao J. Telocytes in cardiac protection. Curr Stem Cell Res Ther 2016; 11 (5): 390-394.

Received June 23, 2017. Accepted July 24, 2017. 